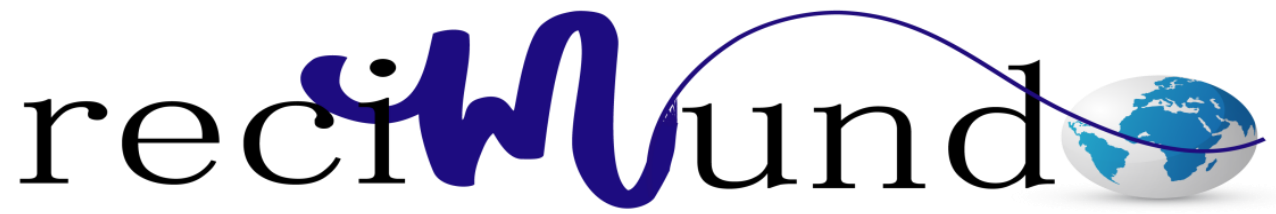

Revista Científica Mundo de la Investigación y el Conocimiento

Katherine Jazmin Arevalo Morales a, Alexandra Silvia Tandazo Lalangui ${ }^{\text {b; }}$ Susana de los Angeles Balarezo Cañar ${ }^{\text {c }}$ Carina Lucía Guadalupe Parraga ${ }^{\text {d }}$

MALNUTRICION ¿enfermedad de los países en desarrollo?

Revista Científica Mundo de la Investigación y el Conocimiento. Vol. 2 núm., 1, febrero, ISSN: 2588-073X, 2018, pp. 446-463

DOI: 10.26820/recimundo/2.1.2018.446-463

Editorial Saberes del Conocimiento

Recibido: 05/12/2017 Aceptado: 10/02/2018

a. Médico Pediatra del área de cuidados intensivos Neonatales del Hospital Doctor Francisco Icaza Bustamante; katykatyarevalo@hotmail.com

b. Médico Pediatra del área de cuidados intensivos Neonatales del Hospital Doctor Francisco Icaza Bustamante; alexadra72@hotmail.com

c. Médico Pediatra del área de cuidados intensivos Neonatales del Hospital Doctor Francisco Icaza Bustamante; susanabalarezoc@hotmail.com

d. Médico Pediatra del área de cuidados intensivos Neonatales del Hospital Doctor Francisco Icaza Bustamante; cary-gp923@hotmail.com 
Vol. 2, núm. 1., (2018)

Katherine Jazmin Arevalo Morales; Alexandra Silvia Tandazo Lalangui; Susana de los Angeles Balarezo Cañar; Carina Lucía Guadalupe Parraga

\section{RESUMEN}

La nutrición es uno de los pilares de la salud y el desarrollo del individuo y por tanto de la sociedad, mientras mejor sea el estado nutricional de un ciudadano más productivo y exitoso será un país. La humanidad está dividida en dos grandes bloques. En uno, se sitúan los que tienen recursos económicos, alimentos suficientes y un sistema sanitario desarrollado que permite cubrir sus necesidades perfectamente; al otro pertenecen todos aquellos que carecen de lo más mínimo para vivir, no cuentan con un sistema sanitario o es precario, no tienen alimentos suficientes en cantidad y la calidad del agua es muy mala. Estas dos situaciones pueden producir malnutrición. El término malnutrición se refiere a las carencias, los excesos y los desequilibrios de la ingesta calórica y de nutrientes de una persona. La mejora de la nutrición empieza por promover la salud materna, lactantes y niños pequeños; reforzar nuestro sistema inmunitario, disminuir las complicaciones durante el embarazo y el parto, reducir el riesgo de sufrir enfermedades no transmisibles (como la diabetes y las enfermedades cardiovasculares) y prolongar la vida. Queda camino por delante para educar a la sociedad en mejorar sus hábitos alimenticios y proveer de alimentos saludables a los pobres.

Palabras clave: Malnutrición, nutrición, países en desarrollo. 


\title{
MALNUTRICION ¿enfermedad de los países en desarrollo?
}

Vol. 2, núm. 1., (2018)

Katherine Jazmin Arevalo Morales; Alexandra Silvia Tandazo Lalangui; Susana de los Angeles Balarezo Cañar; Carina Lucía Guadalupe Parraga

\begin{abstract}
Nutrition is one of the pillars of the health and development of the individual and therefore of society, the better the nutritional status of a more productive and successful citizen will be a country. Humanity is divided into two great blocks. In one, there are those who have economic resources, sufficient food and a developed health system that allows them to cover their needs perfectly; the other belong to those who lack the least to live, do not have a sanitary system or is precarious, do not have enough food in quantity and the quality of the water is very bad. These two situations can produce malnutrition. The term malnutrition refers to the deficiencies, excesses and imbalances of a person's caloric and nutrient intake. Improving nutrition begins by promoting maternal health, infants and young children; strengthen our immune system, reduce complications during pregnancy and childbirth, reduce the risk of non-communicable diseases (such as diabetes and cardiovascular diseases) and prolong life. There is a way ahead to educate society to improve their eating habits and provide healthy food to the poor.
\end{abstract}

Keywords: Malnutrition, nutrition, developing countries. 
Vol. 2, núm. 1., (2018)

Katherine Jazmin Arevalo Morales; Alexandra Silvia Tandazo Lalangui; Susana de los Angeles Balarezo Cañar; Carina Lucía Guadalupe Parraga

\section{Introducción.}

La nutrición es uno de los pilares de la salud y el desarrollo del individuo y por tanto de la sociedad, mientras mejor sea el estado nutricional de un ciudadano más productivo y exitoso será un país. Los niños sanos aprenden mejor. La gente sana es más fuerte, más productiva y está en mejores condiciones de romper el ciclo de pobreza y hambre y de desarrollar al máximo su potencial.

Anteriormente, la desnutrición presente en las sociedades de bajos recursos y en el denominado tercer mundo era el problema a manejar. Desde hace pocas décadas, se asoció otro término: la malnutrición que abarca desde la desnutrición hasta la obesidad.

La humanidad está dividida en dos grandes bloques. En uno, se sitúan los que tienen recursos económicos, alimentos suficientes y un sistema sanitario desarrollado que permite cubrir sus necesidades perfectamente; al otro pertenecen todos aquellos que carecen de lo más mínimo para vivir, no cuentan con un sistema sanitario o es precario, no tienen alimentos suficientes en cantidad y la calidad del agua es muy mala. Estas dos situaciones pueden producir malnutrición.

Unas veces por exceso, como es el caso del bloque desarrollado, en el que la abundancia hace que se coma en exceso alimentos procesados industrialmente, refinados y ricos en grasas saturadas, y donde la obesidad y enfermedades coronarias hacen estragos.

Otras veces por defecto, generalmente en los países subdesarrollados y dependientes de la ayuda exterior, donde el hambre es la que hace estragos causando la muerte y desnutrición en 


\section{MALNUTRICION ¿enfermedad de los países en desarrollo?}

Vol. 2, núm. 1., (2018)

Katherine Jazmin Arevalo Morales; Alexandra Silvia Tandazo Lalangui; Susana de los Angeles Balarezo Cañar; Carina Lucía Guadalupe Parraga

millones de personas cada año, ya que la morbilidad aumenta con la precariedad de estos seres humanos.

Normalmente, la desnutrición y la obesidad se tratan como asuntos independientes que afectan tanto a ricos como a pobres. En realidad, ambas están arraigadas en la pobreza y afectan cada vez más a las mismas comunidades vulnerables. Los países de ingresos medios (incluso algunos de ingresos bajos) son particularmente susceptibles a esta combinación de factores, vinculados a procesos económicos y a cambios en el estilo de vida, lo que da lugar a una dieta excesiva y a enfermedades relacionadas.

Tradicionalmente, las políticas de sanidad y desarrollo en materia de alimentación se han centrado en la desnutrición. Junto con un aumento del gasto público en protección social y salud, estas políticas han erradicado la malnutrición aguda en la mayoría de los países de América Latina y el Caribe y han reducido la malnutrición crónica en un 62\% desde el año 2000. (Nutrición, 2017)

Sin embargo, la deficiencia de micronutrientes combinada con el rápido aumento del sobrepeso, la obesidad y las enfermedades crónicas está convirtiéndose en un problema acuciante en un contexto regional cambiante caracterizado por la alta movilidad geográfica, la urbanización y la transición nutricional.

Según la Organización Mundial de la Salud (OMS) hay un aproximado de 140 millones de personas en América Latina y el Caribe que son afectadas por la malnutrición en exceso. 


\section{MALNUTRICION ¿enfermedad de los países en desarrollo?}

Vol. 2, núm. 1., (2018)

Katherine Jazmin Arevalo Morales; Alexandra Silvia Tandazo Lalangui; Susana de los Angeles Balarezo Cañar; Carina Lucía Guadalupe Parraga

Alrededor de 1900 millones de adultos tienen sobrepeso, mientras que 462 tienen insuficiencia ponderal. Se calcula que 41 millones de niños menores de 5 años tienen sobrepeso o son obesos, hay 52 millones de niños menores de 5 años que presentan emaciación, 17 millones con emaciación grave, y 155 millones sufren retraso del crecimiento.159 millones tienen retraso del crecimiento. (OMS, 2016)

A esa carga hay que sumar los 528 millones de mujeres (29\%) en edad reproductiva que sufren anemia, la mitad de las cuales podría tratase con suplementos de hierro.

Alrededor del $45 \%$ de las muertes de menores de 5 años tienen que ver con la desnutrición. En su mayoría se registran en los países de ingresos bajos y medianos. Al mismo tiempo, en esos países están aumentando las tasas de sobrepeso y obesidad en la niñez. (WHO, 2017.)

Actualmente hay más personas con sobrepeso que con desnutrición en el mundo. El número de personas con sobrepeso ha alcanzado el tope de un billón de personas (de los cuales 300 millones son avisos), comparado con 800 millones malnutridos.

Todos los países del mundo están afectados por una o más formas de malnutrición. Combatir todas las formas de malnutrición es uno de los mayores problemas sanitarios a escala mundial.

Las mujeres, los lactantes, los niños y los adolescentes están particularmente expuestos a la malnutrición. La optimización de la nutrición al comienzo de la vida (en particular durante los 


\section{MALNUTRICION ¿enfermedad de los países en desarrollo?}

Vol. 2, núm. 1., (2018)

Katherine Jazmin Arevalo Morales; Alexandra Silvia Tandazo Lalangui; Susana de los Angeles Balarezo Cañar; Carina Lucía Guadalupe Parraga

1000 días que transcurren entre la concepción y el segundo aniversario del niño) asegura el mejor arranque posible de la vida, con beneficios a largo plazo.

El crecimiento económico, el aumento de la urbanización y los ingresos medios de las personas y la integración de la región en los mercados internacionales, han reducido el consumo de preparaciones tradicionales y aumentado el consumo de productos ultra procesados, un problema que afecta con mayor fuerza a las zonas y países que son importadores netos de alimentos. (Organización Mundial de la Salud, 2017)

Muchas familias no tienen recursos para adquirir suficientes alimentos nutritivos, como frutas y verduras frescas, legumbres, carne o leche, o no tienen acceso a ellos, mientras que los alimentos ricos en grasas, azúcar y sal son más baratos y se pueden adquirir más fácilmente, lo que propicia el rápido aumento del sobre peso y la obesidad entre los niños y adultos, tanto en los países pobres como en los ricos.

Mientras disminuye la desnutrición, la malnutrición por exceso está convirtiéndose rápidamente en una mayor carga para América Latina y el Caribe. La proyección realizada hasta 2078 estima que el sobrepeso y la obesidad generen un costo anual total de 1.000 millones de dólares en Chile, 3.000 millones de dólares en Ecuador y 13.000 millones de dólares en México.

Alimentarse, con todos los tipos de vitaminas, con las proteínas y minerales necesarios, es fundamental para el desarrollo del sistema inmunológico.

Asimismo, factores socio-económicos, genéticos, psicológicos, ambientales y hasta algunos fármacos, son causantes de esta enfermedad que afectan a muchas personas en 


\section{MALNUTRICION ¿enfermedad de los países en desarrollo?}

Vol. 2, núm. 1., (2018)

Katherine Jazmin Arevalo Morales; Alexandra Silvia Tandazo Lalangui; Susana de los Angeles Balarezo Cañar; Carina Lucía Guadalupe Parraga

Latinoamérica y el Caribe, lo que causa afecciones cardiovasculares, ginecológicas, pulmonares, dermatológicas, digestivas, genitourinarias (órganos urinarios y genitales), neurológicas, psicosociales, renales, locomotor, metabólicas y endocrinas.

Todos los países del mundo están afectados por una o más formas de malnutrición. Combatir todas las formas de malnutrición es uno de los mayores problemas sanitarios a escala mundial.

La pobreza multiplica el riesgo de sufrir malnutrición y sus consecuencias. Las personas pobres tienen una mayor probabilidad de sufrir distintas formas de malnutrición. Por su parte, la malnutrición aumenta los costos de la atención de salud, reduce la productividad y frena el crecimiento económico, lo que puede perpetuar el ciclo de pobreza y mala salud.

A nivel mundial, la estrategia de prevención más importante es disminuir la pobreza y mejorar la educación nutricional y las medidas de salud pública.

\section{Definiciones}

MALNUTRICION: es la pérdida de nutrientes suficientes para mantener funcionando de forma sana el organismo y está típicamente asociada con pobreza extrema en países en desarrollo; es una causa común de reducción de la inteligencia en ciertas partes del mundo afectadas por hambruna, es el resultado de una dieta inapropiada, el consumo en excesivo de alimentos o la ausencia de una "dieta balanceada" es frecuentemente observada en países económicamente desarrollados (como indica el incremento de los niveles de obesidad). 


\section{MALNUTRICION ¿enfermedad de los países en desarrollo?}

Vol. 2, núm. 1., (2018)

Katherine Jazmin Arevalo Morales; Alexandra Silvia Tandazo Lalangui; Susana de los Angeles Balarezo Cañar; Carina Lucía Guadalupe Parraga

El término malnutrición se refiere a las carencias, los excesos y los desequilibrios de la ingesta calórica y de nutrientes de una persona: (OMS O. M., 2014)

1) la desnutrición, que incluye la emaciación (un peso insuficiente respecto de la talla), el retraso del crecimiento (una talla insuficiente para la edad) y la insuficiencia ponderal (un peso insuficiente para la edad),

2) la malnutrición relacionada con los micronutrientes, que incluye las carencias de micronutrientes (la falta de vitaminas o minerales importantes) o el exceso de micronutrientes y

3) el sobrepeso, la obesidad y las enfermedades no transmisibles relacionadas con la alimentación (como las cardiopatías, la diabetes y algunos cánceres).

\section{Malnutricion relacionada con micronutrientes}

Las ingestas inadecuadas de vitaminas y minerales (los denominados micronutrientes) se pueden reunir en un mismo grupo. El organismo necesita micronutrientes para producir enzimas, hormonas y otras sustancias esenciales para un crecimiento y desarrollo adecuado.

El yodo, la vitamina A y el hierro son los más importantes en lo que se refiere a la salud pública a escala mundial; sus carencias suponen una importante amenaza para la salud y el desarrollo de las poblaciones de todo el mundo, en particular para los niños y las embarazadas de los países de ingresos bajos. (OMS, Boletín de la Organización Mundial de la Salud. , 2018) 
Vol. 2, núm. 1., (2018)

Katherine Jazmin Arevalo Morales; Alexandra Silvia Tandazo Lalangui; Susana de los Angeles Balarezo Cañar; Carina Lucía Guadalupe Parraga

\section{Sobrepeso y obesidad}

Una persona tiene sobrepeso o es obesa cuando pesa más de lo que corresponde a su altura. Una acumulación anormal o excesiva de grasa puede afectar a la salud.

El índice de masa corporal (IMC) es una relación entre el peso y la altura que se utiliza habitualmente para determinar el sobrepeso y la obesidad en adultos. Se define como el peso de una persona en kilogramos divididos por el cuadrado de la altura en metros $\left(\mathrm{kg} / \mathrm{m}^{2}\right)$. En los adultos, el sobrepeso se define por un IMC igual o superior a 25, y la obesidad por un IMC igual o superior a 30 .

El sobrepeso y la obesidad pueden ser consecuencia de un desequilibrio entre las calorías consumidas (demasiadas) y las calorías gastadas (insuficientes). A escala mundial, las personas cada vez consumen alimentos y bebidas más calóricos (con alto contenido en azúcares y grasas), y tienen una actividad física más reducida.

Ahora bien, la malnutrición por exceso es el consumo de alimentos inadecuados que en el organismo crean un efecto de suma desproporcionada del tejido adiposo, en el cual el peso corporal está por encima de lo ideal, también llamado sobrepeso u obesidad.

DESNUTRICION: la desnutrición es un tipo de malnutrición que se asocia de forma directamente proporcional a la cantidad y la calidad de los nutrientes que se ingieren.

La desnutrición aguda se conoce como la causa directa de la subnutrición (ingesta insuficiente de nutrientes) y es su extensión más crítica. Se traduce en que el cuerpo ya ha 


\section{MALNUTRICION ¿enfermedad de los países en desarrollo?}

Vol. 2, núm. 1., (2018)

Katherine Jazmin Arevalo Morales; Alexandra Silvia Tandazo Lalangui; Susana de los Angeles Balarezo Cañar; Carina Lucía Guadalupe Parraga

gastado sus propias reservas energéticas. Los músculos empiezan a consumirse, el cuerpo se debilita, disminuye el sistema inmunológico y muchos órganos comienzan a fallar.

Existen cuatro tipos principales de desnutrición: emaciación, retraso del crecimiento, insuficiencia ponderal, y carencias de vitaminas y minerales. Por causa de la desnutrición, los niños, en particular, son mucho más vulnerables ante la enfermedad y la muerte.

El retraso del crecimiento impide que los niños desarrollen plenamente su potencial físico y cognitivo.

En los países industrializados, la Desnutrición Calórico Proteica (DCP) es frecuente en adultos mayores institucionalizados (aunque a veces no se sospecha) y en pacientes con trastornos que disminuyen el apetito o alteran la digestión, la absorción o el metabolismo. En los países en desarrollo, la DCP afecta a niños que no consumen suficientes calorías o proteínas. (OMS O. M., 2017)

La DCP puede ser primaria o secundaria. La primaria está causada por una ingestión inadecuada de nutrientes. La DCP secundaria es el resultado de enfermedades o del uso de fármacos que interfieren con el aprovechamiento de los nutrientes. 
MALNUTRICION ¿enfermedad de los países en desarrollo?

Vol. 2, núm. 1., (2018)

Katherine Jazmin Arevalo Morales; Alexandra Silvia Tandazo Lalangui; Susana de los Angeles Balarezo Cañar; Carina Lucía Guadalupe Parraga

Valores que se utilizan con frecuencia para medir la gravedad de la desnutrición calóricoproteica

\begin{tabular}{|l|c|c|c|c|}
\hline \multicolumn{1}{|c|}{ Determinación } & Normal & $\begin{array}{c}\text { Desnutrición } \\
\text { leve }\end{array}$ & $\begin{array}{c}\text { Desnutrición } \\
\text { moderada }\end{array}$ & Desnutrición grave \\
\hline Peso normal (\%) & $90-110$ & $85-90$ & $75-85$ & $<75$ \\
\hline Índice de masa corporal (BMI) & $19-24^{*}$ & $18-18,9$ & $16-17,9$ & $<16$ \\
\hline Albúmina sérica (g/dL) & $3,-5,0$ & $3,1-3,4$ & $2,4-3,0$ & $<2,4$ \\
\hline Transferrina sérica (mg/dL) & $220-400$ & $201-219$ & $150-200$ & $<800$ \\
\hline $\begin{array}{l}\text { Recuento total de linfocitos (por } \\
\mu \mathrm{L})\end{array}$ & $2000-3500$ & $1501-1999$ & $800-1500$ & 0 \\
\hline $\begin{array}{l}\text { Índice de hipersensibilidad } \\
\text { retardada }\end{array}$ & 2 & 2 & 1 & \\
\hline
\end{tabular}

*En los adultos mayores, un IMC $<21$ puede aumentar el riesgo de mortalidad.

$\dagger$ El índice de hipersensibilidad retardada cuantifica la induración provocada por las pruebas cutáneas con antígenos comunes (p. ej., el derivado de especies de Candida o de Trichophyton). Los grados de induración son: $0=<0,5 \mathrm{~cm}, 1=0,5-0,9 \mathrm{~cm}, 2=\geq 1 \mathrm{~cm}$.

Las pruebas de laboratorio son necesarias para identificar las causas cuando se sospecha DCP secundaria. Deben determinarse los niveles de proteína $\mathrm{C}$ reactiva o del receptor de interleucina 2 cuando se desconoce la causa de la desnutrición; estas determinaciones permiten establecer si hay un exceso de citocinas. También pueden realizarse pruebas de función tiroidea.

Es posible detectar anomalías con otras pruebas de laboratorio, que pueden requerir tratamiento. Deben medirse los niveles séricos de electrolitos, nitrógeno ureico, glucosa y, tal vez, 


\section{MALNUTRICION ¿enfermedad de los países en desarrollo?}

Vol. 2, núm. 1., (2018)

Katherine Jazmin Arevalo Morales; Alexandra Silvia Tandazo Lalangui; Susana de los Angeles Balarezo Cañar; Carina Lucía Guadalupe Parraga

calcio, magnesio y fósforo. Los niveles de glucosa en sangre, los electrolitos (sobre todo el potasio y a veces el sodio) y el fósforo, el calcio y el magnesio suelen ser bajos. El nitrógeno ureico suele ser bajo a menos que exista una insuficiencia renal. Puede haber acidosis metabólica. Se solicita un hemograma completo, en el que pueden detectarse anemia normocítica (en general secundaria a deficiencia de proteínas) o anemia microcítica (por deficiencia de hierro).

Deben obtenerse muestras para coprocultivo y pesquisar la presencia de parásitos y huevos si la diarrea es grave o no se resuelve con el tratamiento. En ocasiones, se realizan análisis de orina, urocultivo, hemocultivo, prueba de tuberculina y radiografía de tórax para diagnosticar infecciones ocultas, ya que los individuos con DCP pueden tener una respuesta disminuida a las infecciones.

\section{Causas de malnutricion}

Entre las múltiples causas: la Hambruna, Pobreza, Enfermedades digestivas, Malabsorción, Depresión, Anorexia nerviosa, Bulimia nerviosa, Diabetes no tratada, Síndrome de Wilkie no tratado, Ayuno, Coma, Vegetarianismo estricto, Vegetarianismo, Alcoholismo y la adicción a otras drogas, Consumo excesivo de azúcar y grasas, Sobrepoblación.

Es bastante frecuente encontrar personas desnutridas y con sobrepeso en la misma comunidad o el mismo domicilio, o que una misma persona tenga sobrepeso y a la vez presente, por ejemplo, carencias de micronutrientes.

En varios estudios se han encontrado una relación inversa entre el exceso de grasa corporal y el déficit de nutrientes como hierro, zinc, calcio, vitamina D, vitamina A, ácido fólico, vitamina 


\section{MALNUTRICION ¿enfermedad de los países en desarrollo?}

Vol. 2, núm. 1., (2018)

Katherine Jazmin Arevalo Morales; Alexandra Silvia Tandazo Lalangui; Susana de los Angeles Balarezo Cañar; Carina Lucía Guadalupe Parraga

B12 y sodio. Se viene reportando la coexistencia de la malnutrición por exceso con el déficit de vitaminas y minerales en países desarrollados y en vía de desarrollo.

\section{Consecuencias}

La malnutrición, en cualquiera de sus formas, acarrea riesgos considerables para la salud humana. En la actualidad, el mundo se enfrenta a una doble carga de malnutrición que incluye la desnutrición y la alimentación excesiva con el sobrepeso, sobre todo en los países de ingresos medianos y bajos.

Las investigaciones sobre crecimiento y obesidad muestran que el aumento excesivo en el IMC para la edad después de los 6 meses de edad se asocia con riesgo cardio-metabólico a los 4 años.

Los efectos de la malnutrición, suelen ser irreversibles, sobre todo cuando se dan en individuos que están creciendo o desarrollándose: niños y adolescentes.

Las repercusiones en el desarrollo y las consecuencias de índole económica, social y médica de la carga mundial de la malnutrición son graves y duraderas, para las personas y sus familias, para las comunidades y para los países.

Sin embargo, la deficiencia de micronutrientes combinada con el rápido aumento del sobrepeso, la obesidad y las enfermedades crónicas está convirtiéndose en un problema acuciante en un contexto regional cambiante caracterizado por la alta movilidad geográfica, la urbanización y la transición nutricional 


\section{MALNUTRICION ¿enfermedad de los países en desarrollo?}

Vol. 2, núm. 1., (2018)

Katherine Jazmin Arevalo Morales; Alexandra Silvia Tandazo Lalangui; Susana de los Angeles Balarezo Cañar; Carina Lucía Guadalupe Parraga

Las comidas conocidas como "comida rápida", fueron introducidas aproximadamente desde los años 80's como un sistema de alimentación que explica los cambios del concepto de alimentación tradicional, para una fase industrial de la comida, que además potenciaron la difusión de alimentos cargados de grasas malas y altas calorías, nada saludables.

Todos estos cambios en el patrón de consumo de alimentos, trajeron como consecuencias la aparición de enfermedades crónicas no transmisibles (ECNT) como por ejemplo: cardiovasculares, hipertensión arterial, diabetes tipo II, algunos tipos de cáncer, entre otras afecciones en infantes y adultos. La obesidad como consecuencia de esta forma de alimentación, es una enfermedad multifactorial producida por un desbalance entre la ingesta y la demanda que conlleva a las ECNT inclusive a la muerte.

\section{Materiales y métodos}

La investigación es de tipo bibliográfica, de organizaciones de salud y trabajos de investigación.

\section{Recomendaciones generales}

La mejora de la nutrición empieza por promover la salud materna, lactantes y niños pequeños; reforzar nuestro sistema inmunitario, disminuir las complicaciones durante el embarazo y el parto, reducir el riesgo de sufrir enfermedades no transmisibles (como la diabetes y las enfermedades cardiovasculares) y prolongar la vida. En los niños alimentados con leche materna, se ha demostrado que el riesgo de obesidad en la edad escolar es $15-25 \%$ menor que en los alimentados con fórmulas lácteas. 


\section{MALNUTRICION ¿enfermedad de los países en desarrollo?}

Vol. 2, núm. 1., (2018)

Katherine Jazmin Arevalo Morales; Alexandra Silvia Tandazo Lalangui; Susana de los Angeles Balarezo Cañar; Carina Lucía Guadalupe Parraga

Alimentarse, con todos los tipos de vitaminas, con las proteínas y minerales necesarios, es fundamental para el desarrollo del sistema inmunológico.

El objetivo que hay que lograr es un equilibrio energético y un peso normal, limitar la ingesta de alimentos ricos en grasas y eliminar el consumo de alcohol.

Hacer frente al estado de la malnutrición por exceso, es muy importante para conservar una buena calidad de vida por el mayor tiempo posible, por lo que se debe tener una alimentación acorde y realizar ejercicio físico de forma regular, lo que ayudará a eliminar toxinas (sustancias nocivas para el organismo).

Encabezado por la OMS y la Organización de las Naciones Unidas para la Alimentación y la Agricultura (FAO), el Decenio de las Naciones Unidas de Acción sobre la Nutrición hace un llamamiento para que se adopten políticas en seis esferas de acción: (Morley., 2015.)

1. crear sistemas alimentarios sostenibles y resilientes en favor unas dietas saludables;

2. proporcionar protección social y educación nutricional para todos;

3. armonizar los sistemas de salud y las necesidades de nutrición y proporcionar cobertura universal de las medidas nutricionales esenciales;

4. velar por que las políticas de comercio e inversión mejoren la nutrición;

5. crear entornos seguros y propicios para la nutrición en todas las edades;

6. fortalecer y promover la gobernanza y la rendición de cuentas en materia de nutrición en todos los lugares. 


\section{MALNUTRICION ¿enfermedad de los países en desarrollo?}

Vol. 2, núm. 1., (2018)

Katherine Jazmin Arevalo Morales; Alexandra Silvia Tandazo Lalangui; Susana de los Angeles Balarezo Cañar; Carina Lucía Guadalupe Parraga

\section{Conclusiones}

La malnutrición es una condición que se puede ver en los extremos de nuestra sociedad; tanto en los países desarrollados con el aumento del sobrepeso y obesidad que conlleva a la presencia de enfermedades crónicas no transmisibles.

En los países en desarrollo no se ha erradicado la desnutrición, ahora se asocia la obesidad por la mala calidad de los alimentos (exceso de carbohidratos y grasas saturadas) presentando las mismas enfermedades no transmisibles que los países desarrollados.

Mejorar la calidad de los alimentos, tanto en su preparación como utilizando la suplementacion de micronutrientes.

Incentivar la lectura de la información nutricional de los alimentos y que los fabricantes de alimentos publiquen la verdadera composición de sus productos.

Insistir que la lactancia materna es la mejor "comida" que se puede dar a los niños en sus primeros dos años de vida.

Queda camino por delante para educar a la sociedad en mejorar sus hábitos alimenticios y proveer de alimentos saludables a los pobres. 


\section{MALNUTRICION ¿enfermedad de los países en desarrollo?}

Vol. 2, núm. 1., (2018)

Katherine Jazmin Arevalo Morales; Alexandra Silvia Tandazo Lalangui; Susana de los Angeles Balarezo Cañar; Carina Lucía Guadalupe Parraga

\section{Bibliografía}

Morley., J. (2015.). Desnutrición calórico-proteica. • Obtenido de https://www.msdmanuals.com/es-ve/professional/trastornosnutricionales/desnutrici\%C3\%B3n/desnutrici\%C3\%B3n-cal\%C3\%B3rico-proteica\#.

Nutrición, I. N. (2017). La malnutrición por exceso afecta en un 58\% a América Latina y el Caribe. . Obtenido de http://www.nutrisimon.proyectos.usb.ve

OMS. (2016). La Doble Carga de la Malnutrición: un fenómeno emergente en Salud. Obtenido de https://www.ovsalud.org/.

OMS. (2018). Boletín de la Organización Mundial de la Salud. . 145-224.

OMS, O. M. (2014). Plan de acción mundial para la prevención y el control de las enfermedades no transmisibles 2013-2020,.

OMS, O. M. (2017). Decenio de las Naciones Unidas de Acción sobre la Nutrición 2016-2025.

Organización Mundial de la Salud, O. (2017). La Estrategia Mundial para la Salud de la Mujer, el Niño y el Adolescente 2016-2030. Obtenido de http://www.who.int/es

WHO. (2017.). El costo de la doble carga de la malnutrición. Comisión Económica para América Latina y el Caribe . Obtenido de http://www.who.int/es 